\title{
Intermédialités
}

Histoire et théorie des arts, des lettres et des techniques

Intermediality

History and Theory of the Arts, Literature and Technologies

\section{Présentation. Les potins de Protagoras : pour une sophistique de la transmission}

\section{Éric Méchoulan}

Numéro 5, printemps 2005

Transmettre

Transmitting

URI : https://id.erudit.org/iderudit/1005489ar

DOI : https://doi.org/10.7202/1005489ar

Aller au sommaire du numéro

Éditeur(s)

Centre de recherche sur l'intermédialité

ISSN

1705-8546 (imprimé)

1920-3136 (numérique)

Découvrir la revue

Citer ce document

Méchoulan, É. (2005). Présentation. Les potins de Protagoras : pour une sophistique de la transmission. Intermédialités / Intermediality, (5), 9-20. https://doi.org/10.7202/1005489ar 


\title{
Présentation. \\ Les potins de Protagoras: \\ pour une sophistique \\ de la transmission
}

\author{
Éric Méchoulan
}

L

orsque le mot «présentation » est affiché au début d'un numéro de revue, il passe naturellement inaperçu. Quoi de plus évident que d'écrire quelques pages pour mettre rapidement sous les yeux des lecteurs les points importants des articles ici réunis? Il ne s'agit guère que d'introduire à ce qui sera transmis. Pourtant, la question même de la présentation doit être posée. On pourrait raconter une fable, partir d'un potin, décrire un schéma, écrire sous forme de propositions logiques, recourir à l'étymologie, prendre un ton docte, adopter l'allure de la conversation, favoriser l'aphorisme, chercher de soigneuses images. Chacun de ces choix pose un problème de transmission. Selon le sujet dont je dois traiter et le lecteur que je suppose, mais aussi en fonction des supports de la transmission (qu'ils soient matériels, comme le papier ou l'écran, ou institutionnels, comme le fait d'écrire ici dans une revue «savante»), les possibilités ne sont pas si nombreuses: en l'occurrence, je pourrais à la limite partir d'un potin, non m'y arrêter; je pourrais glisser quelques aphorismes, mais certainement pas m'en contenter; la conversation ne m'est pas vraiment permise, le dialogue autorisé avec suspicion; quant au style figuré, je ne dois pas exagérer, car le lecteur savant se méfie d'images qui semblent rendre trop séduisante l'austérité scientifique (Buffon déjà passait pour un zoologue douteux tant il écrivait bien), comme s'il fallait que l'écriture savante s'installât à cinquante mètres sous la surface de la mer, là où les courants turbulents et l'écume éparpillée de l'écriture s'évanouissent dans le calme plat des profondeurs de la réflexion. 
La bibliographie matérielle a suffisamment montré comment les formes de publication pouvaient infléchir les réceptions et participer à la production de sens des œuvres pour que la «profondeur» remonte en fait à la surface ${ }^{1}$. De même que la perspective en peinture permet des effets de profondeur, les appareils du discours savant autorisent les effets de sens. Cela ne signifie pas que l'on ne sorte jamais des illusions, mais que la transmission de la vérité est partie prenante de la vérité de la transmission (ce chiasme contribuant lui aussi à une certaine logique de la réflexion qui aime à retourner les évidences dans la rapidité d'une formule). C'est pourquoi je préfère ici poser des problèmes généraux à partir d'un cas particulier plutôt que de thématiser les enjeux immédiatement dans leur plus grande largeur.

\section{LA GRÂCE DE LA PRÉSENTATION}

Ainsi, lorsque Socrate demande à Protagoras de lui montrer en quoi la vertu peut être enseignée et transmise, en quoi la politique est une technique dont il serait possible de faire l'apprentissage, le sophiste lui répond-il en proposant au choix un mythe ou une explication:

- Si donc tu es en état de nous démontrer [epideixai] plus clairement que la vertu peut s'enseigner, ne nous refuse pas cette démonstration [epideixon].

- Je ne te la refuserai pas, Socrate; mais voulez-vous que je vous la présente [epideixo], vieillard parlant à des jeunes gens, sous la forme d'un mythe, ou sous celle d'un discours explicatif?

Beaucoup des auditeurs lui répondirent de faire comme il voudrait. «Eh bien, dit-il, il me semble qu'un mythe sera plus agréable [chariesteron, plus gracieux]².»

Protagoras évalue la situation, offre des modes de présentation différents, et quand il lui faut choisir, élit le mythe sans doute pour mieux s'inscrire dans la différence d'âge qu'il a d'office fait remarquer («vieillard parlant à des jeunes gens»). Mais la grâce (charis) est aussi une des composantes importantes de la relation politique.

Pour les Grecs, la grâce est une conséquence de la liberté : le citoyen qui parvient à une autonomie domestique peut consacrer son loisir aux affaires publiques. À la manière des nobles, il entre dans des relations qui ne sont pas

1. Voir Donald McKenzie, Bibliography and the Sociology of Texts, Londres, The British Library, coll. "The Panizzi Lectures », 1986.

2. Platon, Protagoras, trad. Alfred Croiset, introduction et notes de Pierre-Marie Morel, Paris, Éditions Les Belles Lettres, 1997, p. 37-39, 320b 7 - c 7. 
tenues simplement par le besoin de vivre ensemble, mais par les bonnes grâces et les belles actions. Comme le souligne Aristote, « une cité est la communauté des lignages et des villages menant une vie parfaite et autarcique. C'est cela, selon nous, mener une vie bienheureuse et belle. Il faut donc poser que c'est en vue des belles actions qu'existe la communauté politique, et non en vue de vivre ensemble ${ }^{3}$. Émile Benveniste a montré combien la grâce suppose plus la gratuité d'une prestation que l'obligation d'un retour et d'une contre-prestation ${ }^{4}$. Cette grâce souveraine, chez les Anciens, n'offre pas de caractère absolu. Chacun doit savoir en user pour que l'ordre de la polis fonctionne au mieux. Ainsi, le terme de charis désigne aussi bien

la grâce, la faveur avec tous ses dons et complaisances, que la reconnaissance qui lui est due; il embrasse tout le domaine de la largesse, de la prévenance et de la réciprocité, ainsi que la façon agréable, amène et gracieuse de se comporter entre donateur et bénéficiaire. Nous n’avons pas d'équivalent rigoureux pour ce mot, encore que charis corresponde sensiblement à ce que recouvrent les verbes plaire et complaire 5 .

La grâce ordonne un lien social qui passe moins par la supériorité de la force que par la puissance de la générosité. Elle fait de la société un locus amœenus, un lieu amène, où l'amitié l'emporte sur la rivalité. Christian Meier a signalé l'importance proprement politique de la grâce dans un moment où la noblesse grecque perd de son pouvoir effectif: «Lorsque, au cours des siècles mouvementés de l'époque archaïque, l'autorité de la tradition cessa une bonne fois d'être une évidence incontestable, les nobles ne surent pas toujours s'adapter à la vie collective ${ }^{6}$ ", avec pour résultat la mise en place de régimes démocratiques où les nobles jouent, cependant, un rôle primordial, quant aux modes de gestion de la cité, aux manières d'argumenter et de juger des événements, aux styles de vie et de langage. La grâce est la puissance des faibles ${ }^{7}$. En liaison

3. Aristote, Politiques, trad. Pierre Pellegrin, Paris, Éditions Garnier-Flammarion, 1993, III, 9, 128ob 42-1281a 3.

4. Émile Benveniste, Le vocabulaire des institutions indo-européennes, Paris, Éditions de Minuit, 1969, vol. I, p. 201-202.

5. Christian Meier, La politique et la grâce: anthropologie politique de la beauté grecque, trad. Paul Veyne, Paris, Éditions du Seuil, coll. «Des travaux », 1987 [1985], p. 37.

6. Christian Meier, La politique et la grâce, p. $5^{8}$.

7. Aristophane (Les nuées, 112) et Aristote (Rhétorique, 1402a 29-30) soulignent que Protagoras enseignait justement comment faire en sorte que le plus faible de deux arguments l'emporte sur le plus fort. 
avec l'aidos (la pudeur, la honte, le respect), la grâce cherche la conciliation plutôt que l'affrontement, l'émulation des générosités plutôt que les guerres intestines. C'est pourquoi elle est, pour beaucoup, un art de la parole, aidée en cela par Peitho (la persuasion). La grâce ne peut se réduire à quelques banalités esthétisantes, elle compose un des modes fondamentaux des relations politiques (jusqu'au «charisme» problématique de certains tyrans).

Quand Protagoras choisit donc le mythe en raison de son caractère «plus gracieux» pour les jeunes gens, il montre immédiatement, dans son mode de présentation même, comment la vertu du lien politique peut être transmise. À la question de Socrate qui lui réclame une présentation argumentée du fait que la vertu politique serait transmissible et enseignable, Protagoras répond déjà implicitement par la grâce offerte d'un choix, puis par l'élection d'un mythe. Le mode de présentation ne vient pas en plus, au contraire il fait pleinement partie de l'argument de Protagoras.

\section{L'APPRENTISSAge de LA VERTU}

Comment le sophiste justifie-t-il au bout du compte que la vertu politique relève bien d'une technique et fasse l'objet d'une transmission, même si les meilleurs politiques comme Démosthène semblent incapables de faire de leurs propres enfants des hommes aux vertus aussi éminentes que les leurs (ainsi que Socrate le fait remarquer)?

Protagoras fait d'abord une comparaison imaginaire qui nous permet de mieux réaliser ce que chacun a littéralement sous les yeux:

Supposons que la cité ne pût subsister sans que nous fussions tous des flûtistes, chacun dans la mesure où il en serait capable; que cet art aussi fût enseigné à tous et à tous publiquement et dans le privé [kai touto kai idia kai demosia], qu'on châtiât quiconque jouerait faux, et qu'on ne refusât cet enseignement à personne, de même qu'aujourd'hui la justice et les lois [nomimon, règles, prescriptions conformes à l'usage] sont enseignées à tous sans réserve et sans mystère, à la différence des autres métiers - car nous nous rendons service réciproquement, j’imagine, par notre respect de la justice et de la vertu, et c'est pour cela que tous sont toujours prêts à révéler et à enseigner la justice et les lois [nomima] -, eh bien, dans ces conditions, $[\ldots]$ est-ce que tu crois, Socrate, me dit-il, qu'on verrait plus souvent les fils des bons flûtistes l'emporter sur ceux des mauvais? Je n'en crois rien quant à moi, mais je pense que celui qui aurait le fils le mieux doué pour la flûte le verrait se distinguer. (Protagoras, 327a 4- b 9)

Bien évidemment, tout n’est pas seulement une affaire de don personnel. Protagoras avait auparavant fait remarquer que l'éducation commence au plus 
jeune âge, mais les riches ont l'avantage d'envoyer leurs enfants à l'école plus longtemps. Le sophiste ne se fait aucune illusion sur les modes de reproduction des savoirs et sur les qualités des jeunes gens: celles-ci dépendent sans doute de leurs talents naturels, elles résultent aussi de l'enseignement qu'on leur a procuré. Cependant, l'important relève de la teneur quotidienne et parfois implicite de l'apprentissage. Il faut parfois décrire et expliquer telle pratique ou tel usage, mais l'exemple même de tous ceux qui entourent le jeune garçon puis l'adolescent et l'adulte offre d'office des actualisations des règles communes. Toute la réciprocité sociale des usages forme les jeunes autant qu'elle se (per)forme dans les services mutuels les plus simples: indications, éclaircissements, remarques, critiques ou corrections. Ce sont, bien entendu, toutes ces médiations qui servent à transmettre les justes usages de la communauté, mais elles sont transmises de façon immédiate.

Protagoras utilise ensuite une autre comparaison encore plus évidente: "c'est comme si tu cherchais le maître qui nous a enseigné à parler le grec [hellenizein]»(Protagoras, 327e 2 - 328a 1). En fait, l'enfant baigne dans sa langue maternelle comme il baigne dans les formes de vie sociales. C'est - là encore implicitement - indiqué par les différentes significations du verbe hellenizein: parler grec, parler correctement, voire parler tout simplement, mais aussi être Grec, être un homme digne de ce nom, voire être un homme tout court. L'apprentissage quotidien de la langue maternelle est en même temps une éducation civique et un enseignement moral. La médiation du langage est immédiatement appropriation des règles communes. À l'instar de nombre de tribus dans le vaste monde qui se désignent elles-mêmes d'un nom spécifique qui renvoie en même temps au fait d'être un homme, les Hellènes trouvent dans le foyer d'un seul nom leur différence d'avec les barbaroï et leur identité d'êtres humains.

On doit noter que tout le dialogue recourt de façon obsessionnelle à la quête du juste nom et de la bonne désignation, c'est un dialogue onomastique, comme s'il mettait en œuvre la valeur instructive et immédiatement sociale des nominations. D'autant qu'à ces noms sont aussi associés des effets d'étrangeté (Pittacos a été «nourri dans une langue barbare» (Protagoras, 341c 9) et Prodicos venant de Céos est chargé de donner les significations des termes de Simonide, qui diffèrent du grec d'Athènes) qui font que la question de la transmission passe aussi par la problématique de la traduction. Dès le début du dialogue, l'ami de Socrate s'étonne d'apprendre qu'une personne a plus retenu l'attention de Socrate que le bel Alcibiade pour lequel il éprouve des sentiments 
si clairs et il lui demande : "asto è xéno", un citoyen ou un étranger? Le terme d'astos désigne d'abord généralement le «citadin", avant de renvoyer dans la langue du droit athénien au fait d'être «citoyen" même si l'on habite dans une bourgade éloignée de la ville 8 . Comme pour hellenizein, valeurs générique et identitaire se chevauchent. Or, la personne savante qui a paru plus belle encore que le jeune Alcibiade aux yeux de Socrate est bien un étranger: Protagoras d'Abdère. Et, de plus, un étranger qui en amène d'autres avec lui et qui menace d'entraîner ailleurs de jeunes Athéniens qui voudraient le suivre dans ses pérégrinations. L'enjeu civique (peut-on apprendre la vertu?) recoupe l'enjeu identitaire (que font ces étrangers à la jeunesse athénienne? Quels modèles de vie apportent-ils avec eux? Quelle valeur de la migration (et non pas même de l'immigration) supposent-ils?).

Cependant, le tort manifeste de Socrate est de s'en remettre trop simplement aux noms eux-mêmes, alors que Protagoras cherche des modes de présentation. Celui-là croit au dictionnaire des idées, celui-ci à la grammaire de la vie commune9 ${ }^{9}$ Le nom devrait permettre de reconnaître l'identité de celui qui a droit de cité, la grammaire régule les formes de présentation dans lesquelles les idées circulent et prennent sens dans la cité. Il faut l'étranger d'Abdère pour faire apercevoir au parfait Athénien qu'est Socrate (lui qui se targuera lors de son procès de n'être jamais sorti du territoire d'Athènes que pour combattre des ennemis) que l'apprentissage de la vertu et la technique politique des amitiés sont affaire d'éducation et ne relèvent pas simplement d'une naturalité (spécifiquement athénienne).

Il est, en effet, caractéristique de ce dialogue de Platon que Protagoras y apparaisse à l'occasion malmené par la verve socratique, réticent à poursuivre la joute et doive avouer à la fin qu'il s'est contredit, mais également que Socrate semble sous le charme du sophiste, pris lui aussi dans certaines contradictions, se lançant dans un long discours alors qu'il avait lui-même réclamé un dialogue à réponses brèves, adoptant des pratiques quasi-sophistiques, et concluant à l'étonnement général que leurs positions respectives ont rigoureusement pivoté:

8. Voir Louis Gernet, Droit et institutions en Grèce antique, Paris, Éditions Flammarion, coll. "Champs", 1982 [1968], p. 268.

9. Protagoras était aussi connu pour ses travaux de grammairien et son souci de la propriété de l'expression. 
Or, il me semble que notre discours [logos] même, en arrivant à sa conclusion, devient comme notre accusateur et se moque de nous, et que, s'il pouvait prendre la parole [phonèn], il nous dirait: «Vous êtes de plaisants personnages, Socrate et Protagoras: toi, Socrate, qui niais que la vertu pût s'enseigner, voici que tu mets tous tes efforts à te contredire en démontrant que tout est science, la justice, la tempérance, le courage, ce qui est le plus sûr moyen qu'on peut enseigner la vertu [...]. D'autre part, Protagoras, qui avait d'abord mis en fait qu'elle se pouvait enseigner, semble maintenant s'appliquer à se contredire.»(Protagoras, 361a $4-\mathrm{c} 3$ )

On pourrait certes remarquer que l'apprentissage de la vertu passait, pour Protagoras, par la technè alors que, pour Socrate, la vertu est enseignable parce qu'elle relève d'une épistémè, d'un savoir. Les deux positions ne sont donc pas strictement identiques. Mais il faut aussi reconnaître que c'est Socrate lui-même qui parle d'abord de la technè politikè avant de glisser de l'apprentissage de la vie politique à l'enseignement de la vertu et que le sophiste ne fait que reprendre là ses propres indications. Tout au plus Protagoras présente-t-il dans le dialogue comment il sait «rendre les autres habiles à parler [legein] » (Protagoras, 312d 6-7). Legein signifie aussi: mettre ensemble, recueillir, rassembler, compter, ranger, en plus de dire, célébrer ou penser. L'art du sophiste consiste justement à recueillir et ranger les appareils de pensée afin de les faire servir à bon escient, en parvenant à transmettre aux jeunes gens ou aux citoyens les formes mêmes de la transmission que représente toute véritable politique. Une cité, un pays où l'on ne transmet plus la langue avec toutes ses subtilités et ses histoires parfois conflictuelles avec les autres langues ou les accents différents, où on ne transmet plus les héritages et les formes de vie avec leurs logiques immanentes et leurs rapports à d'autres configurations culturelles, risque bien de tourner très vite court.

\section{Écriture, oralité ET MÉTAPHYSIQUe}

Dans le mythe de Protagoras, Hermès, petit dieu des messages, des carrefours et du commerce, transmet aux hommes, de la part de Zeus, les moyens de mettre ensemble, de faire parler dans les désaccords et les concordes possibles tous les citoyens, également savants dans l'art du politique, mais plus ou moins experts dans la vertu politique. Une fois que les hommes savent comment se mettre ensemble, alors ils peuvent transmettre à d'autres hommes, comme le fait Protagoras, comment exercer pleinement leur mission de citoyens. Même Socrate qui semblait au départ si réticent à l'admettre reconnaît à la fin du dialogue que 
Protagoras, sans l'avoir directement convaincu, paraît bien lui avoir «transmis » sa position ${ }^{10}$.

Mais qu'en est-il de la transmission même du dialogue? Socrate le rapporte à un ami, un de ses hetairos, mettant donc en scène d'entrée de jeu les modes usuels de transmission des nouvelles, des discours, des événements à Athènes. On pourrait croire que Platon élève ainsi le commérage à la dignité de la dialectique, s'il ne fallait penser à l'inverse que la philosophie platonicienne trouve d'autant plus de crédibilité dans l'univers antique qu'elle repose souvent sur la transmission la plus habituelle des potins ${ }^{11}$. En même temps, comme pour tout exercice de commérage, il faut affirmer la valeur du sien, d'où toute une lutte pour écarter les autres sources de potins : c'est ainsi qu'une théorie des

10. On peut noter que, dans la transmission du «texte» du Protagoras (je mets des guillemets car il s'agit là, bien entendu, d'une notion propre aux temps modernes qui fait l'économie des configurations anciennes de production des œuvres et de leur transmission sur lesquelles je ne peux m’étendre ici), Al-Fârâbî, au x $\mathrm{x}^{\mathrm{e}}$ siècle, tend à durcir l'opposition Socrate-Protagoras en repliant le Protagoras sous le Théétète où Socrate critique radicalement la conception du sophiste selon laquelle «l'homme est la mesure de toutes choses » (Al-Fârâbî, La philosophie de Platon, ses parties, l'ordre de ses parties, du commencement jusqu'à la fin, trad. de l'arabe et annoté par Olivier Dedeyn et Nassim Lévy, Paris, Éditions Allia, 2002, p. 10-11); par contre, Marsile Ficin, son premier traducteur en latin (en 1468), confond à ce point les positions qu'il attribue directement à Platon le mythe de Protagoras (voir sa Theologia platonica de immortalitate animorum, Raymond Marcel (éd.), Paris, Éditions Les Belles Lettres, 1964, livre XIV, chapitre 10, vol. II, p. 289290) et Castiglione, dans son ouvrage promis à une immense influence dans le procès de civilisation des mœurs, reprend le mythe sans prendre la peine de l'attribuer à l'un ou à l'autre malgré la méfiance des humanistes pour les sophistes (voir Baldassar Castiglione, Le livre du courtisan, présenté et traduit par Alain Pons d'après la version de Gabriel Chapuis (1580), Paris, Éditions Flammarion, 1991 [1528], p. 335).

11. On peut rappeler, par exemple, que le Banquet est un récit fait par Apollodore de Phalère à des amis et des connaissances; or, Apollodore commence par évoquer une rencontre quelques jours plus tôt avec une de ses connaissances qui lui avait justement demandé de lui rapporter ce qui s'était passé en cette fameuse soirée entre Agathon, Alcibiade et Socrate, car il en avait entendu parler par quelqu'un qui le tenait de Phénix, le fils de Philippe, mais qui ne connaissait pas tous les détails et qui lui avait affirmé que lui, Apollodore, pourrait les lui donner et, de fait, Apollodore en a eu le récit par un certain Aristodème, un petit homme qui allait toujours nu-pieds et qui était un des amoureux de Socrate... toute la structure traditionnelle du désir de potin lance ainsi sur la piste de l'amour. 
Idées peut s'affirmer contre les opinions du vulgaire ou doit même contraindre les opinions à apparaître comme opinion, autrement dit comme une forme parmi d'autres d'énoncés possibles et comme une forme qui ne concerne que les apparences.

Il faut rappeler qu'Athènes, à la différence de nombreuses cités grecques, ne possède guère d'institutions par où la tradition et l'histoire de la ville sont assurées et transmises de génération en génération: pas de mnêmones, d'experts en souvenir comme tels. L'éloge funéraire (epitaphios) était l'occasion de rappeler les hauts faits du passé, mais c'est à peu près la seule tradition officielle. La transmission se faisait immédiatement dans les exemples, les récits, les figures que mobilisaient les orateurs dans les assemblées de la boulè où étaient prises les décisions collectives ${ }^{12}$. Il faut attendre la toute fin du $\mathrm{v}^{\mathrm{e}}$ siècle pour que des «archives» soit conservées dans le Metroon, que ce soit sous forme de papyrus, de tablettes ou de stèles gravées. Mais cela ne signifie pas que l'on puisse retrouver là une idée de copie préservée pour les temps à venir qui permettrait de transmettre le contenu d'un original. Il est caractéristique que ce que nous appellerions des copies aient autant, parfois plus d'autorité que les «originaux». Sans compter que ces copies ne cherchent pas l'exactitude que nous en réclamons. Même à l'écrit, les variations évidentes de l'oralité l'emportent. Car la tradition est éminemment orale: le potin est la forme par excellence de la transmission, une technique collective de l'art politique. Platon y décèle une façon de présenter sa philosophie, de la mettre à l'épreuve immédiate de sa transmission. C'est pourquoi on ne saurait trop prêter attention aux mises en scène des dialogues, aux lieux, aux interlocuteurs, aux récits.

Quand Socrate réclame de Protagoras une "présentation " (epideixis) de sa proposition initiale, Platon en orchestre bien toute l'importance, au point de mettre soigneusement en scène ses propres dialogues. Pourtant le fait d'écrire cette présentation, de la transmettre ainsi par la fixation d'un texte archivable plutôt que remémoré ne va-t-elle pas exactement à l'encontre de tout le propos platonicien, puisque, comme il le dit ou plutôt l'écrit à un moment, «les livres ne peuvent ni répondre ni interroger» (Protagoras, 329a 3-4)?

12. «But the democratic polis traditions of the assembly went side by side with any other gossip, rumour, anecdote or historical example that orators also chose to use.» (Rosalind Thomas, Oral Tradition and Written Record in Classical Athens, Cambridge, Cambridge University Press, 1992, p. 199) 
C'est le sophiste Protagoras qui semble imposer un modèle écrit plutôt que le philosophe Platon. Pourtant, même si Protagoras prend comme exemple d'éducation immédiate de la vertu politique la lecture ${ }^{13}$, on voit bien la présence encore mixte du déchiffrage de l'écriture, de la vocalisation nécessaire et de la mémorisation ${ }^{14}$. Néanmoins, le modelage des citoyens par les lois suit, pour Protagoras, l'exemple de l'apprentissage de l'écriture:

[...] de même que le maître d'écriture, pour les enfants qui ne savent pas encore écrire, trace d'abord les lettres avec son stylet et leur remet ensuite la page où ils devront suivre docilement l'esquisse des lettres, ainsi la cité, traçant à l'avance le texte des lois, œuvre des bons et anciens législateurs, oblige ceux qui commandent et ceux qui obéissent à s’y conformer. (Protagoras, 326d 2-d 8)

Il s'agit là de mettre en valeur, non les appropriations éventuelles des lecteurs, mais la technique machinale de la soumission aux lois.

C'est exactement cette technique que critiquera Isocrate lorsque, un demisiècle après le passage de Protagoras et avant la création de l'Académie, il installe à Athènes son école et rédige entre autres un ouvrage intitulé Contre les sophistes. Il y affirme qu'ils "prétendent lui [l'élève] transmettre la science du discours aussi facilement que la connaissance des lettres de l'alphabet ${ }^{15}$. » Or, pour Isocrate comme pour Platon, c'est la transmission orale que l'on doit préserver avant tout, y compris par une institutionnalisation bien pensée de la lecture. Celui qui a écrit n'est plus là pour répondre aux questions ou aux

13. "Quand les enfants, sachant leurs lettres, sont en état de comprendre les paroles écrites $[. .$.$] , il fait lire à la classe, rangée sur les bancs, les vers des grands poètes et lui$ fait apprendre par cœur ces œuvres remplies de bons conseils, et aussi de digressions, d'éloges où sont exaltés les antiques héros, afin que l'enfant, pris d'émulation, les imite. » (Protagoras, 325e $2-326$ a 3 )

14. De même Plutarque, dans ses Vies des dix orateurs, nous dit que Lycurgue fut le premier, au IV siècle, à imposer que les tragédies d'Eschyle, d'Euripide et de Sophocle fussent conservées en koinô (en commun), sans doute dans le Metroon, afin que ceux qui voulaient en rejouer les pièces puissent et doivent les consulter, mais il précise que les pièces devaient alors être lues à voix haute (grammatéa paranaginoskein) aux acteurs qui allaient les jouer : l'écrit doit passer par une vocalisation officielle et une mémorisation de la part des acteurs. Voir Vie de Lycurgue, dans Euvres morales, trad. Marcel Cuvigny, Paris, Éditions Les Belles Lettres, 1981, t. XII, 841f 8-12.

15. Isocrate, "Contre les sophistes", dans Cinq discours, édition, introduction et commentaire de Robert Flacelière, Paris, Presses universitaires de France, coll. «Érasme », 1961, p. 86. 
critiques: le discours écrit est sans père pour en protéger et en révéler la vérité. Comment faire en sorte de transmettre un écrit qui puisse toujours être protégé, qui puisse «répondre» comme de lui-même?

Il ne faut pas oublier que, si Platon a légué de nombreux dialogues à la postérité, il a aussi laissé derrière lui l'Académie. Autrement dit, toute une école où sont transmis, non seulement les textes eux-mêmes, mais aussi la tradition de leur interprétation. D’une génération l'autre, le sens des œuvres est maintenu: chacun en devient le père et le nouveau protecteur, sous l'autorité de celui qu'on appelait le diadokhos (le «successeur »). L'auteur est un principe de regroupement des textes, comme le disait Michel Foucault, mais il est également celui qui fait autorité pour en recomposer les significations et les enjeux. L’Académie est l'autorité platonicienne par excellence, celle qui défend les justes interprétations de ces textes qui roulent ici et là, dans des contextes imprévisibles, en touchant des lecteurs inconnus. On ne peut dire que Platon ait mal joué, dans la mesure où l'Académie a quand même duré presque un millénaire ${ }^{16}$ : quelle institution pourrait aujourd'hui se prévaloir d'une telle durée?

L'oralité mise en scène dans les dialogues platoniciens constitue ainsi à la fois une récupération des procédures traditionnelles de mise en sens et une appropriation de nouveaux supports intellectuels : l'écriture, d'un côté, l'institution académique, de l'autre. C'est ainsi que le support social du commérage dans la cité peut trouver une autre dimension historique dans le compagnonnage du sens que produit l'Académie - compagnonnage dont la formation demeure fondée sur le lien pédérastique ${ }^{17}$ : l'institution du sens passe aussi par

16. Fondée vers 385 avant Jésus Christ, c'est en l'année 529 de notre ère que l'empereur Justinien décida de fermer l'Académie pour des raisons qui tenaient plus de la xénophobie que du rejet de la philosophie.

17. Voir Henri-Irénée Marrou, Histoire de l'éducation dans l'Antiquité, Paris, Éditions du Seuil, coll. «Points. Histoire», 1981 [1948], p. 64. Je rappelle (sans pouvoir m'y étendre ici) qu'un des modèles classiques, chez les Grecs comme chez les Latins, pour comprendre la relation entre un auteur et un lecteur est celui du rapport entre l'éraste et l'éromène, qu'il prenne la forme d'une entreprise de séduction (le lecteur sera qualifié de kalos, beau) ou de rapport de puissance et d'autorité (le lecteur est alors un katapugôn, sodomisé). C'est pourquoi ce sont en général les esclaves qui lisent à voix haute les ouvrages, car la lecture est une preuve de "passivité». Aristote est connu pour être un des rares à lire lui-même les nombreux ouvrages dont il était, par ailleurs, collectionneur. Voir Jesper Svenbro, Phrasikleia: anthropologie de la lecture en Grèce ancienne, Paris, Éditions La Découverte, 1988. 
une institution du sexe, car ce sont à tous ces niveaux qu'il faut penser ce qu'est «transmettre».

Dans une certaine mesure, on peut même voir émerger la philosophie de ce moment particulier de l'histoire grecque où les formes et les usages de la transmission devenaient fort problématiques: la métaphysique pourrait alors passer pour une appropriation écrite du potin. Au moment où les «maîtres de vérité $^{18}$ " ne peuvent plus opérer selon les dispositifs oraux et mémoriaux traditionnels, la sophistique et la philosophie proposent des solutions à la question sociale et politique de la transmission - la métaphysique qui pose la question de ce qu'est l'être au creux de ses apparitions n'apparaît que mieux mise en jeu par les problèmes de transmission. La polis antique est avant tout un espace d'apparitions où les hommes ne sont pas de simples objets du monde, mais parlent et agissent en tant qu'hommes: ce sont les rituels techniques du langage, des gestes, des danses qui rythment les manières d'apparaître et les façons de transmettre. L'être apparaît en fait au foyer de ce qu'il transmet et qui est reçu.

Nous avons beau avoir multiplié depuis la fin du XIX siècle les dépassements de la métaphysique, il semble que nos nouvelles techniques, qu'elles concernent la politique ou la transmission, ne nous aient pas véritablement sorti de ses antiques problèmes. Il serait même possible de voir le développement récent de nos enquêtes intermédiales comme l'utile résurgence de cette question originaire de la transmission. Les analyses ici proposées pourraient alors être diffusées comme des potins: «saviez-vous que l'intermédialité est la métaphysique ramenée à sa fabrique ancienne?...»

18. Voir Marcel Détienne, Les mâttres de vérité dans la Grèce archaïque, Paris, Éditions La Découverte, 1990 [1967]. 